\title{
UV-photochemistry of the biologically relevant thiol group and the disulfide bond: Evolution of early photoproducts from picosecond X-ray absorption spectroscopy at the sulfur K-Edge
}

\author{
Miguel Ochmann ${ }^{1}$, Abid Hussain ${ }^{1}$, Inga von Ahnen ${ }^{1}$, Amy A. Cordones ${ }^{2}$, Kiryong Hong ${ }^{3}$, \\ Jae Hyuk $\mathrm{Lee}^{2}$, Rory $\mathrm{Ma}^{3}$, Katrin Adamczyk ${ }^{1}$, Tae Kyu $\mathrm{Kim}^{3}$, Robert W. Schoenlein ${ }^{2}$, \\ Oriol Vendrell ${ }^{4}$, and Nils Huse ${ }^{1, *}$ \\ ${ }^{1}$ Department of Physics, University of Hamburg and Max Planck Institute for the Structure and \\ Dynamics of Matter, Center for Free Electron Laser Science, 22761 Hamburg, Germany \\ ${ }^{2}$ Ultrafast X-ray Science Lab, Chemical Sciences Division, Lawrence Berkeley National Laboratory, \\ Berkeley, California 94720 \\ ${ }^{3}$ Department of Chemistry and Chemistry Institute of Functional Materials, Pusan National \\ University, Busan 46241, South Korea \\ ${ }^{4}$ Center for Free-Electron Laser Science, DESY and The Hamburg Centre for Ultrafast Imaging, \\ 22607 Hamburg, Germany
}

\begin{abstract}
We report on the UV-induced photochemistry of the biologically relevant sulfur-containing thiol group and the disulfide bond in solution using picosecond X-ray absorption spectroscopy at the sulfur K-edge. This study provides element-specific insight into the $267-\mathrm{nm}$ induced photo-chemistry of two model compounds, an aromatic thiol and an aliphatic disulfide. Our transient spectra point to two primary and several secondary photoproducts, and our analysis may aid in understanding UV damage in proteins.
\end{abstract}

Sulfur is of great importance in all fields of chemistry ranging from material science to biochemistry due to its electronic versatility and high natural abundance. It is found in many proteins either in the active site of enzymes as metal-sulfur-cluster or as part of the protein backbone in form of the two amino acids methionine or cysteine, relevant in the protein biosynthesis and important for protein tertiary structure, respectively. Cysteines form very stable disulfide bonds which may also acts as a radiation shield to protect the protein from structural changes due to harmful radiation. Additionally, the thiol form of cysteine also takes part in many repair mechanism such as thiol-disulfide exchange reactions. Investigations of the photochemistry of the structural motifs of the thiol group and the disulfide bond are therefore of greater general interested and our spectroscopic approach provides a route to element-specific information.

While most ultrafast optical and to some extent vibrational methods are limited in either their information content (due to broad and nondescript features) or in their

* Corresponding author: nils.huse@uni-hamburg.de 
applicability to solvated and/or large systems, time-resolved X-ray absorption spectroscopy (TRXAS) provides a versatile probe for local electronic and chemical environments. We have investigated two model compounds in solution with TRXAS at the sulfur K-edge to establish the early timescale photochemistry of the thiol group and the disulfide bond and address long-standing open questions.
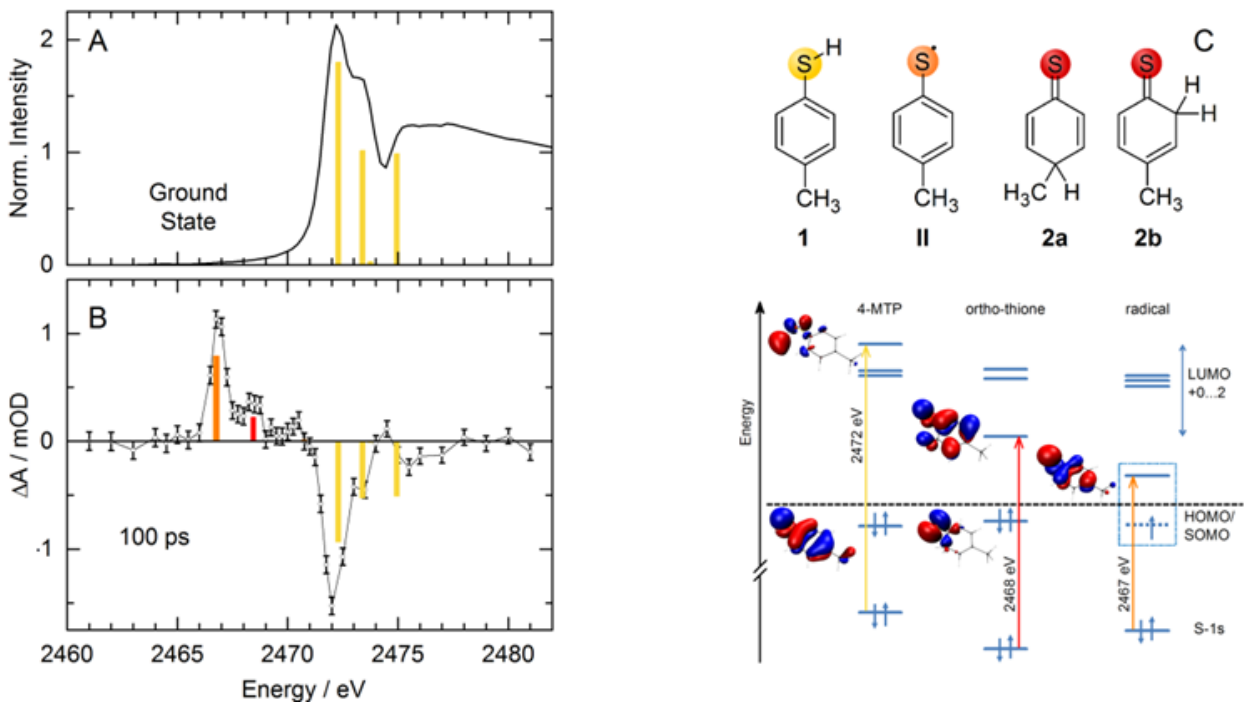

Fig. 1. A. Static sulfur-1s spectrum of 4MTP. B. Corresponding pump-probe spectrum at 100 ps. The identified species are shown in panel $\mathbf{C}$ along with their lowest transitions and the corresponding relevant frontier orbitals.

We investigated an aromatic thiol (4-MTP, 1) which has previously been studied by the Bradforth and Ashfold groups using ultrafast optical, vibrational, and TKER spectroscopy [1-3]. Homolytic bond cleavage of the S-H group produces arylthiyl and hydrogen radicals upon UV illumination and a signature of another transient photoproduct which emerges within the first 50 ps and coincides with a partial decay of the thiyl radical population. While these studies where not fully conclusive towards identifying the secondary photoproduct, sulfur-!s transitions show the transient XAS signature of the arylthiyl radical $\mathbf{I}$ and unambiguously identify the secondary photoproduct as the two thione isomers $\mathbf{2 a}$ and $\mathbf{2} \mathbf{b}$ of the parent molecule $\mathbf{1}$, in support of the interpretations of previous work [4]. Fig. 1A shows the static XAS spectrum of 1 with lowest RASSCF calculated transitions (yellow). In Fig. 1B the 100ps differential spectrum reveals the photoproducts (II orange, $\mathbf{2} \mathbf{a}$ and $\mathbf{2 b}$ red).

We furthermore investigated the simplest organic disulfide (DMDS, 3), a model for the biologically very relevant disulfide bond motif. Time-resolved optical studies $[5,6]$ reported two UV-induced reactions, S-S and C-S bond dissociation. By employing 267 $\mathrm{nm}$ TRXAS at the sulfur K-edge, we identified both, S-S and C-S bond dissociation, producing thiyl and perthiyl radicals (II and III). We observed the partially irreversible decay of these photoproducts, with the perthiyl radical decaying more rapidly than the thiyl radical, an observation which might have added to the confusion in previous literature. Our results point to the accessibility of C-S bond dissociation at $267 \mathrm{~nm}$ wavelength. We also identified traces of secondary decay products of the perthiyl radical, disulfur (3), thioformaldehyd (4) and hydrogen sulfide radicals (IV). Figure 2 shows our experimental data and summarizes our spectral analysis. 

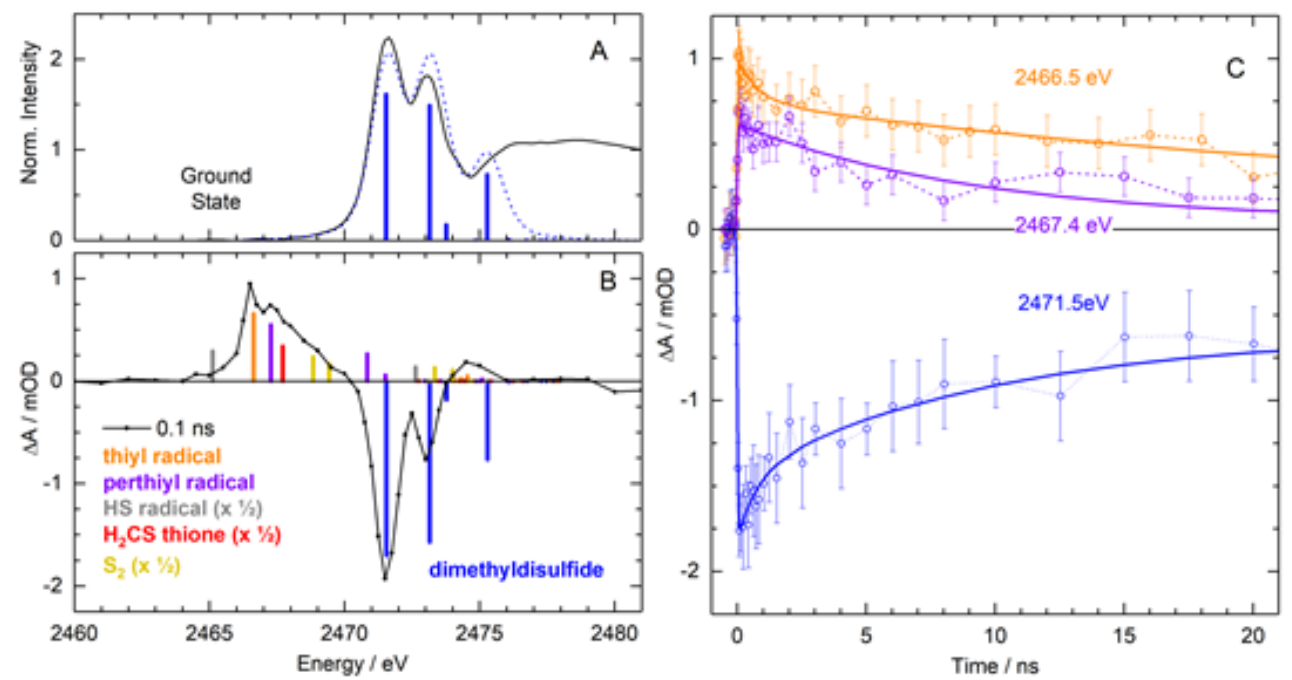

Fig. 2. A. Static XAS spectrum of 2 with the RASSCF calculated bound-bound transitions (blue). B. Differential spectrum at 100 ps delay with lowest RASSCF transitions of transient photoproducts (II orange, III purple, $\mathbf{3}$ yellow, $\mathbf{4}$ red and IV grey). C. Transients and fits of exponential decays [7].

In summary, we have gained new insight from TRXAS at the sulfur K-edge into the formation of photoproducts of two biologically highly relevant sulfur groups in solution, and our studies pave the way to femtosecond investigations at XFELs.

This work was supported by the Director, Office of Science, Office of Basic Energy Sciences, the Chemical Sciences, Geo-sciences, and Biosciences Division under the Department of Energy, Contract DE-AC02-05CH1 1231 (AC, KH, JL, RWS), by the National Research Foundation of Korea (grants 2007-0056095, 2013S1A2A2035406, 2013R1A1A2009575, 2014R1A4A1001690), in part by the Global Research Laboratory Program (grant 2009-00439), and the Max Planck POSTECH/ KOREA Research Initiative Program (grant 2011-0031558) through the National Research Foundation of Korea (TKK KH). MO, IvA, KA, and NH acknowledge funding from the Max Planck Society. MO and $\mathrm{NH}$ also acknowledge funding from the Deutsche Forschungsgemeinschaft (SFB 925, project A4). This research used resources of the Advanced Light Source, a DOE Office of Science User Facility. The authors thank Bruce Rude for his continued support of beamline hardware.

1. T. A. A. Oliver, Y. Zhang, M. N. R. Ashfold, S. E. Bradforth, Faraday Discuss. 150, 439-458 (2011)

2. Y. Zhang, T. A. A. Oliver, M. N. R. Ashfold, S. E. Bradforth, Faraday Discuss. 157, 141- 163 (2012)

3. D. Murdock, S. J. Harris, T. N. V. Karsili, G. M. Greetham, I. P. Clark, M. Towrie, A. J. Orr-Ewing, M. N. R. Ashfold, J. Phys. Chem. Lett. 3, 3715- 3720 (2012)

4. M. Ochmann, I. von Ahnen, A. A. Cordones, A. Hussain, J. H. Lee, K. Hong, K. Adamczyk, O. Vendrell, T.K. Kim, R.W. Schoenlein, N. Huse, J. Am. Chem. Soc. 139, 4797- 4804 (2017)

5. A. Rinker, C. D. Halleman, M. R. Wedlock, Chem. Phys. Lett. 414, 505-508 (2005).

6. C. W. Bookwalter, D. L. Zoller, P. L. Ross, M. V. Johnston, J. Am. Soc. Mass Spectrom. 6, 872-876 (1995).

7. M. Ochmann, A. Hussain, I. von Ahnen, A. A. Cordones, K. Hong, J. H. Lee, R. Ma, K. Adamczyk, O. Vendrell, T. K. Kim, R. W. Schoenlein, N. Huse, J. Am. Chem. Soc. 140, 6554-6561 (2018) 